\title{
Algumas considerações metodológicas sobre os estudos epidemiológicos das Lesões por Esforços Repetitivos (LER)
}

\author{
Methodological issues in epidemiological \\ studies of Repetitive Strain Injuries (RSI)
}

Serafim Barbosa Santos Filho 1,2

Sandhi Maria Barreto 3

\footnotetext{
1 Departamento de Medicina Preventiva e Social, Faculdade de Medicina, Universidade Federal de Minas Gerais. Av. Alfredo Balena 190, Belo Horizonte, MG 30110-100, Brasil. 2 Secretaria de Saúde de Belo Horizonte. Av. Pedro II 307, Belo Horizonte, MG 30710-010, Brasil. 3 Laboratório de Epidemiologia e Antropologia Médica, Centro de Pesquisa René Rachou, Fundação Oswaldo Cruz. Av. Augusto de Lima 1715, Belo Horizonte, MG 30190-002, Brasil.
}

Abstract Repetitivestrain injuries (RSI) area major public health problem with social and economic repercussions. This article presents a critical review of the published literature on RSI. The vast majority of the studies conducted in the last two decades were cross-sectional and exploratory. Results are difficult to interpret due to such methodological problems as lack of standardization and accuracy in identification of cases, incl usion of cases with potentially different diseases, varying levels of severity in the same study, lack of distinction between prevalent and incident cases, lack of precision in the definition and measurement of exposure, and confounding, besides the built-in constraint of cross-sectional studies for inferring causality. Some of these problems result from our insufficient knowledge of upper-li mb soft ti ssue disorders and the absence of reliable diagnostic tests. Such problems could be addressed by studies whose design considered and stratified cases according to certainty and specificity of diagnosis. Key words Occupational Diseases; Cross-Sectional Studies; Epidemiology

Resumo As lesões por esforços repetitivos (LER) constituem-se um problema de saúde pública, com repercussões sociais e econômicas. Este artigo apresenta uma revisão crítica dos estu dos epidemi ológi cos das últimas duas décadas. A grande mai oria desses estudos foi de corte transversal e exploratório, e os resultados são difíceis de interpretar devido aos problemas metodológicos. Entre os principais problemas notados estão: falta de padronização e rigor na defi ni ção e identificação de casos, não diferenciação dos "casos" segundo a especi fici dade e gravidade clínica, inclusão de "casos" prevalentes e inci dentes no mesmo estudo, impreci são e precariedade na defi nição e mensuração dos fatores de exposi ção e de confusão rel evantes, além das limi tações intrínsecas dos estudos de prevalência para inferir causal i dade. Essas li mi tações resultam em parte da insufi ciência de conhecimento científico das lesões de tecidos moles de membros superiores e da falta de métodos diagnósti cos confiáveis. Entretanto, tais dificuldades podem ser mi nimizadas com desen hos mais a propriados e que levem em consi deração e estratifi quem casos segundo a especificidade do quadro clíni co e grau de certeza do diagnóstico.

Palavras-chave Doenças Ocupacionais; Estudos Transversais; Epi demiologia 


\section{Introdução}

A nomenclatura Lesões por Esforços Repetitivos (LER) começou a ser utilizada no final da década de 50, para designar um conjunto de patologias, síndromes e/ ou sintomas músculoesqueléticos que acometem particularmente os membros superiores, relacionando-seo seu surgimento ao processo de trabalho (Browne et al., 1984; McDermott, 1986; Sommerich et al., 1993; Assunção \& Rocha, 1993; Assunção, 1995).

O olhar mais dirigido para esses quadros ocorreu inicialmente a partir do acometimento de trabalhadores de determinados ramos de atividade, observado com maior destaque em alguns países do mundo. Primeiramente, descreveu-se a "occupational cervicobrachial disorder" em operadores de caixa registradora, perfuradores de cartão e datilógrafos japoneses; na Austrália, descreveu-se a “occupational overuse injury" em digitadores e trabalhadores de linha de montagem; posteriormente foram referidos casos de "cumulative trauma disorders" em trabal hadores expostos a traumas cumulativos, incluindo operadores de terminais de vídeo, nos Estados Unidos da América (McDermott, 1986; Kiesler \& Finholt, 1988; Assunção \& Rocha, 1993; Assunção, 1995; Ong, 1994).

A partir desses marcos referenciais, o fenômeno tem sido relatado em vários países, incluindo a América Latina, onde o Brasil vem-se destacando por crescentes estatísticas de prevalências nas agências de seguro social e serviços de saúde (Nusat/INSS, 1995; Pinheiro et al., 1995; Santos Filho, 1995; Vorcaro, 1995).

Do ponto de vista da questão central trazida no corpo da conceituação do problema, isto é, a possível associação com o processo de trabalho, as nomenclaturas utilizadas têm sido tomadas como equivalentes, sendo mais empregada a expressão Lesões por Esforços Repetitivos, traduzindo a terminologia "RepetitiveStrain Injuries", utilizada pela primeira vez na Austrália (Assunção \& Rocha, 1993).

Atual mente observam-se inúmeros levantamentos mostrando a dimensão do fenômeno enquanto problema de saúde e econômico-social. Para isso, basta remeter-se às estatísticas mundiais e nacionais de custos e afastamentos temporários ou definitivos da força de trabalho, com diagnósticos de acometimentos de membros superiores (McDermott, 1986; Kiesler \& Finholt, 1988; Sommerich et al., 1993; Williams \& Westmorland, 1994; Ross, 1994; Bammer, 1996). Os diagnósticos englobam desde sintomas músculo-esqueléticos inespecíficos até estágios variados de patologias bem definidas e já descritas nos compêndios de reumatologia e ortopedia.
Não obstante a dimensão social do problema, o que tem preponderado do ponto de vista científico é a dificuldade e controvérsia na caracterização dos quadros chamados de LER, refletindo as limitações da prática médica diária para lidar com a questão. Isso, tanto no que se refere à caracterização dos quadros clínicos englobados nessa denominação, quanto aos aspectos envolvidos na sua causalidade e história natural.

Este artigo apresenta uma revisão crítica da literatura recente sobre as LER, discutindo as limitações dos estudos realizados e levantando algumas questões metodológicas importantes para as investigações futuras. A principal base de dados consultada foi o Sistema Medline, sendo também consideradas referências bibliográficas citadas em livros e artigos sobre o assunto. Na seleção, foram observados título, resumo e tipo de desenho epidemiológico, estando mencionados aqui, em ordem cronológica, parte dos artigos que foram encontrados nas bibliotecas pesquisadas (Sistema Bireme), priorizando-se a busca de publicações mais recentes.

Prevalências e fatores de risco destacados na literatura

Ferguson (1971) conduziu um dos primeiros levantamentos em telegrafistas, estudando 93\% dos trabalhadores do serviço público de telecomunicações da Austrália, e encontrou taxas de prevalência de 14\% para cãibra e 5\% para dores musculares nos membros superiores. Ao comparar esses "casos" com o restante do grupo de trabalhadores que não apresentaram sintomas, o autor encontrou associações significativas com fatores da organização e condições de trabalho e outros, como insatisfação e instabilidade no emprego, conflitos com supervisor e dificuldade de adaptação ao posto e equipamentos de trabalho $(p<0,005)$. Nesse estudo também encontrou-se uma associação altamente significativa $(p<0,001)$ entre a presença de cãibra/dores musculares e algum tipo de manifestação neurótica grave, sem, entretanto, esclarecer a influência do trabal ho como possível fator de sobrecarga psíquica.

Luopajarvi et al. (1979) compararam prevalências de sintomas como dor e tensão muscular nos diversos segmentos superiores (pescoço, ombro, cotovelo e punho) em trabalhadoras de linha de montagem (embaladoras) e balconistas finlandesas, estas últimas sendo consideradas como não expostas a movimentos repetitivos. Encontraram maiores prevalên- 
cias desses sintomas entre as embaladoras $(p<$ 0,001 ), sendo que nesta categoria os sintomas também foram de maior gravidade $(p<0,001)$. Entre as embaladoras as partes do corpo mais afetadas foram as mãos (53\%), o pescoço (37\%) e os ombros ( $9 \%$ ). No estudo foram utilizados como fontes de dados, tanto entrevista clínica, quanto exames médico e fisioterápico, ampliando o espectro de validação dos achados. Entre os resultados também verificou-se que o número de embaladoras que havia procurado médico para aquele problema no último ano foi maior do que o de balconistas $(p<0,001)$, reforçando a coerência dos achados. Embora tenham sido identificados os movimentos característicos da atividade de trabalho, o estudo não aprofunda em avaliações sobre o risco associado a esses movimentos específicos e outros fatores concorrentes.

Maeda et al. (1982) estudaram operadores de máquina registradora no Japão e construíram, a partir de uma abordagem ergonômica, uma matriz correlacionando queixas de fadiga nos membros superiores a posturas assumidas pelos segmentos corporais envolvidos. Esse procedimento permitiu identificar, como fatores significativos relacionados às queixas nas diversas regiões do corpo, posturas forçadas do tronco e ombro, inclinação da cabeça e pescoço e desvio ulnar forçado.

Hocking (1987) estudou o setor de telecomunicações na Austrália, entre 1981 e 85, e encontrou taxas de prevalências de lesões dos membros superiores que variavam de 3,3\% a $34 \%$ entre telegrafistas, datilógrafos, telefonistas e outros trabalhadores administrativos, sendo que $76 \%$ dos casos identificados resultaram em afastamentos do trabalho. Outros estudos no mesmo setor, com a utilização de exame clínico para confirmação diagnóstica, apontaram prevalência de $19,5 \%$ de doenças músculo-esqueléticas dos membros superiores entre telefonistas (Hocking, 1987).

Armstrong et al. (1987) relataram prevalência de $4,4 \%$ de tendinites, detectadas através de entrevista e exame clínico em trabalhadores americanos de sete ramos de atividade, cujos movimentos foram agrupados de acordo com gradiente de força e repetitividade. $\mathrm{O}$ autor encontrou uma associação estatisticamente significativa entre a ocorrência de tendinites e uso de força excessiva e alta repetitividade no trabalho, comparando-se com baixo emprego de força e pouca repetitividade no desempenho das tarefas $(p \varangle 0,001)$. Após ajustamento por setor de atividade, foi obtido um "odds ratio" de 29,4, mas o intervalo de confiança não foi mencionado. Nesse estudo, delimitou-se criteriosa- mente a identificação, tanto de casos, como de caracterização dos movimentos de esforço e repetição.

Nos anos 90 predominam ainda os inquéritos exploratórios, mas despontaram algumas iniciativas de investigações mais elaboradas. Higgs et al. (1993) procuraram descrever a influência da idade sobre os quadros de LER, lembrando a importância desse fator na gênese de al gumas patologias ósteo-musculares degenerativas. Os autores lembram que em relação à síndrome do túnel do carpo, alguns estudos mostraram pico de incidência entre 35 e 45 anos, bem como aumento de alterações el etromiográficas com a idade. No estudo de Higgs e colaboradores, em trabalhadores americanos, não houve diferença significativa entre os grupos etários, nem em relação ao gênero. Foram incluídos trabalhadores de ramos de atividade diferentes, estratificados por faixa etária e definidos critérios de casos baseados em relatos e exame físico. O processo de avaliação deu-se o mais "cego" possível, para não influenciar os resultados do estudo.

Vanderpool et al. (1993) acharam prevalência de 57\% de sintomas de síndrome do túnel do carpo entre ecocardiografistas americanos. No entanto, as limitações do estudo, inclusive quanto à amostragem obtida, não permitem conclusões significativas. Chiang et al. (1993) encontraram prevalências de 30,9\% de doenças do ombro e pescoço, $15 \%$ de epicondilite e $14,5 \%$ de síndrome do túnel do carpo em trabalhadores de processamento de peixes de indústrias tailandesas, índices semelhantes a outros achados de tenossinovites na indústria alimentícia. A estimativa de risco (OR) ajustada para alguns fatores mostrou que, além da relação com esforço e repetitividade, a síndrome do túnel do carpo está mais associada com o sexo feminino ( $O R=2,6$; IC =1,3-5,2) e também ao uso de contraceptivo oral $(\mathrm{OR}=2 ; \mathrm{IC}=1,2$ $5,4)$. No estudo foram utilizados como fontes de dados entrevistas, exame físico e análise de tarefas, classificando os trabal hadores de acordo com riscos ergonômicos, combinando grau de esforço e repetitividade. Entre os critérios de definição de casos, foram levados em conta todos os possíveis diagnósticos diferenciais.

Franzblau et al. (1993) estudaram a freqüência de sintomas de ombro e pescoço em trabaIhadores de artes e ilustrações de um escritório médico nos Estados Unidos, sendo que entre eles os artistas gráficos tiveram mais sintomas de extremidades distais do membro superior. Nessa categoria observaram-se mais horas de uso de computador e maior utilização do mouse. No estudo foram realizados observação de 
campo, entrevista, exame médico padronizado e eletroneuromiografia, seguindo-se protocolo de critérios de caso. A eletromiografia não mostrou alterações significativas entre as categorias estudadas, mas os parâmetros para interpretação desse exame foram questionados, como em outros estudos. Apesar da investigação ter-se orientado por fontes de dados diversificadas e criteriosas, o estudo é limitado quanto ao tamanho da amostra, e também não se esclarece exatamente em que aspectos os grupos comparados são diferentes, em termos de processo de trabalho.

Westgaard et al. (1993) encontraram prevalências de $77 \%$ e $74 \%$ de sintomas nos membros superiores em trabalhadores noruegueses, da produção alimentícia e de serviços administrativos, respectivamente, sobressaindo as queixas de ombro e pescoço, atribuídas à maior sobrecarga nessas regiões. Foram estudados trabalhadores do ramo da produção e do setor terciário, agrupados de acordo com carga de trabal ho e outros fatores de risco por região anatômica. O autor ampliou a coleta de informações, caracterizando a atividade e a sobrecarga ocupacional e extra-ocupacional, porém o tamanho e o método de seleção da amostra dificultam a análise e interpretação dos resultados.

Bernard et al. (1994) encontraram prevalência de $41 \%$ de sintomas músculo-esqueléticos de membros superiores em trabal hadores de terminais de vídeo de empresa jornalística americana, predominando queixas no ombro e pescoço. Ao se comparar com os trabalhadores não acometidos, foram encontradas na análise multivariada associações significativas com aspectos da organização do trabalho, como: exigência de produção ( $O R=1,5$; IC =1,0-2,2), ausência de participação nas decisões sobre o trabalho (OR =1,6; IC =1,2-2,1), ausência de suporte de supervisão ( $O R=1,4$; IC =1,2-2,5) e extensão da jornada de operação do terminal, este último apresentando gradiente dose-resposta. O estudo foi realizado em 973 trabalhadores, representando 93\% da amostra aleatória inicial.

Ohlsson et al. (1995) compararam prevalências em mulheres expostas e não-expostas a atividades repetitivas na indústria de equipamentos elétricos da Suécia, coletando dados a partir de entrevistas, exame físico, análise dos postos de trabalho e exames laboratoriais. Doenças do ombro-pescoço, epicondilites e lesões de mão foram associadas aos seguintes fatores: atividade repetitiva ( $O R=4,6$; IC =1,912 ), idade mais avançada (54 anos ou mais) $(\mathrm{OR}=1,9 ; \mathrm{IC}=1,0-3,5)$, pagamento por produti- vidade $(O R=4,9 ; I C=1,5-16)$, tendência à tensão muscular ( $O R=2,3$; IC $=1,3-4,9$ ) e estresse ( $O R=1,9$; IC =1,1-3,5). Outros fatores importantes foram satisfação no trabalho, esforço de concentração e concomitância de sintomas psicossomáticos, bem como movimentos e posturas assumidos na tarefa. O autor ressalta o achado de sintomas mais significativos relacionados a tempo de serviço inferior a dez anos ( $O R=9,6$; IC =2,8-33). Ao interpretar esse resultado, questiona se esse fato poderia ser devido à elevada média de idade dos participantes do estudo. Apesar dos critérios bem definidos de coleta e diagnóstico, a amostra do estudo foi pequena ( 79 trabalhadoras expostas).

English et al. (1995), em um estudo exploratório do tipo caso-controle, encontraram associação entre lesões de membros superiores e movimentos específicos realizados em diferentes atividades de trabalho. Os casos e controles foram selecionados em clínicas ortopédicas, sendo o diagnóstico mais freqüente entre os casos a síndrome do túnel do carpo. Foram coletados dados sobre hábitos pessoais e história ocupacional recente (até dois anos). Como fatores significativos, encontraram-se movimentos de prensa digital, manutenção de postura fixa e em desvio, flexão e extensão dos segmentos, rotação e abdução dos ombros e jornada ininterrupta do movimento repetitivo. A magnitude de associação desses movimentos com lesões de mão, punho, cotovelo e ombros variou de $1,3(I C=1,13-1,49)$ a 5,1 (IC = 2,02$12,83)$. Sexo feminino esteve associado à lesão de punho e antebraço ( $O R=1,75$; IC $=1,07$ 2,85), mas não à síndrome do túnel do carpo. As categorias de maior risco foram: cabeleireiras, faxineiras, secretárias, trabalhadoras de linha de montagem, operadoras de máquinas em geral, professores, açougueiros e el etricistas. Os autores não informam sobre diagnósticos e critérios de inclusão dos controles no estudo.

Vários estudos de série de casos e outros descritivos foram realizados nas décadas de 80 e 90. Nessas décadas também foram feitas tentativas de sistematizar os resultados dos levantamentos existentes, principalmente no que concerne aos fatores relacionados ao trabalho. Browne (1984) reuniu os principais fatores associados ao surgimento e agravamento dos quadros em a) fatores biomecânicos, como contrações musculares prolongadas e posturas inadequadas, freqüência e força empregada no movimento repetitivo, inadequações do posto de trabalho, desenho e manutenção dos equipamentos e tensão muscular associada a estresse; b) fatores da organização do trabalho, 
como ausência de pausas, incentivo à produtividade, falta de treinamento e supervisão inadequada; e c) aspectos agravantes, como o despreparo, negligência, pressões econômicas e outros, dos profissionais de saúde, retardando diagnósticos ou intervindo inadequadamente. De acordo com Kiesler \& Finholt (1988), Sommerich et al. (1993) eWilliams \& Westmorland (1994), os seguintes fatores ocupacionais estariam associados à presença de sintomas nos membros superiores: características posturais assumidas no trabalho, equipamentos inadequados, ausência de pausas durante a jornada, insatisfação no trabalho e treinamentos inadequados. Além desses, os autores relacionaram fatores não-ocupacionais, como pouco tempo de lazer; características demográficas, como sexo, estado civil e filhos; e hábitos pessoais, como prática de esportes. Somam-se aos fatores ocupacionais já relatados os estresses mecânicos localizados, movimentos vibratórios, temperaturas frias e outros que conformariam quatro categorias, de acordo com Putz-Anderson (Sommerich et al., 1993): nível de esforço empregado, quantidade e freqüência da atividade repetitiva, postura e tempo de repouso.

Bammer (1988, 1989a, 1989b, 1990, 1996), procurando alinhavar os estudos feitos sobre as Lesões por Esforços Repetitivos, reuniu investigações cuja análise tivesse utilizado técnicas multivariadas. Entre os achados mais significativos, destacou os fatores relacionados à organização do trabalho, como as pressões de tempo e produtividade, monotonia e grau de autonomia sobre o que faz. Com relação ao papel de fatores não diretamente relacionados ao trabalho, como idade e aspectos psicossociais, bem como ao papel dos componentes biomecânicos, os resultados foram bastante variados e as associações estatísticas frágeis. Exceção deve ser feita para as posturas assumidas pelos segmentos corporais no desempenho das atividades de trabalho. A autora refere ainda as principais associações verificadas por categorias específicas nos estudos de prevalência, sendo elas: dentistas e doenças cervicais e do ombro; embaladoras e caixas e síndrome do desfiladeiro torácico; operadores de terminais de vídeo e síndromes no pescoço; soldadores de estaleiro e síndrome do impacto.

Quanto aos sintomas avaliados, Bammer mostra que a direção das pesquisas pode ser reunida em três grandes grupos: os relacionados a sintomas gerais, como dor, parestesias e redução de força, sendo tratados como uma única "entidade" e chamados de LER, doença cervicobraquial ou doenças por traumas cumulativos. O segundo grupo aborda as doen- ças específicas, como síndrome do túnel do carpo, por exemplo, havendo poucos estudos e mostrando prevalências menores em relação ao anterior. O terceiro grupo concentra-se sobre sintomas em região/ segmento anatômico, mostrando maiores prevalências para doenças do ombro e pescoço.

Stock (1991), recorrendo à meta-análise, fez o levantamento dos fatores associados aos quadros de LER, atribuindo destaque àqueles cujos estudos foram realizados com maior rigor metodológico. O autor avaliou a associação entre fatores ergonômicos e doenças músculoesqueléticas de pescoço e membros superiores, levando-se em conta a qualidade dos estudos, em termos de população amostral, parâmetros de classificação de casos, de exposição e não-exposição, instrumentos de coleta de dados e método de avaliação. Utilizou extensa base de dados oficiais e outros não indexadose identificou 54 trabalhos potencial mente relevantes, dos quais apenas três preencheram os critérios definidos, embora limitados pelo desenho de corte transversal. As conclusões apontaram fortes evidências de associação entre trabalho repetitivo e de esforço e doenças de mãos e punhos. Entre estas, a síndrome do túnel do carpo aparece associada com repetição e esforço, com um odds ratio de 15,5 (IC = 1,7141,5) após ajustamento.

\section{Discussão}

Não obstante os resultados encontrados até o momento, os elementos destacados na revisão conduzida por Stock (1991) podem ser tomados para al gumas observações de ordem metodológica em relação aos estudos sobre as Lesões por Esforços Repetitivos. Essas observações servem para mostrar as dificuldades na compreensão desses quadros, sendo que o que caracteriza tal dificuldade no esclarecimento das "doenças" e do nexo causal com o trabalho deve-se, em grande parte, à própria insuficiência de conhecimento e/ou inadequação dos métodos de investigação. Isto é, além da escassez de estudos, as pesquisas realizadas apresentam problemas de ordens diversas.

As principais limitações dos estudos epidemiológicos sobre as LER poderiam ser resumidas em cinco pontos: falta de padronização e rigor na definição e identificação de casos; não diferenciação dos "casos" segundo a especificidade e gravidade clínica; inclusão de "casos" prevalentes e incidentes no mesmo estudo; imprecisão e precariedade na definição e mensuração dos fatores de exposição e de confusão 
relevantes; limitação metodológica dos desenhos adotados. Além disso é importante ressaltar a falta de estudos epidemiológicos para avaliação do efeito dos procedimentos de prevenção e tratamentos adotados na prática médica até então.

As LER, diferentemente de outras patologias ocupacionais como as pneumoconioses, por exemplo, não constituem uma entidade clínica específica, mas abrangem inúmeros quadros sintomáticos e síndromes, al guns difusos e outros bem delimitados anatômica e/ ou fisiopatologicamente. Apesar de fazerem parte de um grupo único de doenças ósteo-musculares, ao se agruparem quadros difusos e bem definidos, corre-se o risco de incluir entre os casos um percentual de falso-positivos e também doenças e sintomas com gêneses distintas. A inclusão de falsos positivos e de patologias distintas no mesmo estudo tenderiam a diluir ou mesmo distorcer as associações investigadas.

Como na maioria dos processos crônicodegenerativos e psicossociais, vários aspectos certamente contribuem para a produção das LER. É possível que haja alguns fatores comuns associados à ocorrência das síndromes e sintomas agrupados sob a denominação LER (como sexo, por exemplo). Entretanto, o conhecimento de fatores tão gerais contribui muito pouco para esclarecer e melhorar o conhecimento sobre esses sintomas e síndromes além de não auxiliar a discussão de medidas preventivas específicas.

Por outro lado, os quadros músculo-esqueléticos de membros superiores não são exclusivamente ocupacionais, podendo ter sua gênese associada também a outros fatores como esporte, postura fora do trabalho, traumas prévios, repouso, fatores psicossociais e outros. $\mathrm{O}$ que parece ter crescido na atualidade é a fração de casos potencialmente relacionada ao trabalho. Portanto, seria importante no estudo de um quadro clínico como a síndrome do túnel do carpo, tentar identificar os fatores ocupacionais e extra-ocupacionais específicos e dimensionar a importância de cada um desses fatores para a ocorrência da doença na população de estudo. Claro que tal abordagem só seria possível se a definição de caso não levasse em conta a própria exposição a movimentos repetitivos no trabalho, pois isso enviesaria o resultado do estudo.

Predominam na literatura investigações de corte transversal, em que doença e característica ou exposição são estudados concomitantemente. Nestes estudos os grupos "expostos" ou "casos" são comparados a trabalhadores de outro ramo de atividade ("não expostos") ou sem sintomas ("controles"). Falta nesses estudos a explicitação dos critérios de elegibilidade para seleção do grupo "não exposto" ou “controle", dificultando avaliar a validade dos resultados obtidos.

Como se sabe, as doenças de início insidioso e lento podem levar a uma série de medidas e atitudes, muitas vezes imperceptíveis, que modificam a história recente do doente, levando a erros de interpretação conhecidos como causalidade reversa. Certos fatores aparentemente "causadores" da doença podem na verdade refletir respostas adaptativas do organismo a processos ósteo-musculares instalados insidiosamente. No caso das LER, o estudo de casos prevalentes, com duração variada, pode levar a erros importantes na identificação de fatores ocupacionais e não-ocupacionais. Observa-se, por exemplo, que ao sentir dores difusas ou inespecíficas em um determinado segmento do corpo, o indivíduo começa também a evitar certos movimentos e mesmo modificar sua maneira de andar, sentar, deitar, carregar objetos, chegando a abolir certas atividades para minimizar essas dores, à medida que o processo mórbido progride. Devido a essa característica insi diosa e lenta, pode tornarse difícil a identificação do momento de aparecimento dos sintomas, bem como a precisão em relação às suas posturas anteriores ao problema. Sendo assim, a falta de abordagem da questão temporal na grande maioria dos estudos conduzidos dificulta muito a avaliação da real implicação dos fatores identificados na produção das doenças estudadas. Por outro lado, não é possível eliminar a possibilidade de uma implicação dessas "medidas adaptativas" do organismo na própria evolução da doença, pois muitas vezes essas adaptações podem levar a uma piora na postura ou sobrecarga de outros grupos musculares. Com isso, é fundamental que os estudos epidemiológicos levem em conta o início e duração tanto da doença quanto da exposição, bem como mudanças ocorridas no decorrer do processo, inclusive aquelas relativas ao término da exposição, caso o trabalhador tenha mudado de função ou emprego. Idealmente, quanto mais recente o diagnóstico, menor seria o risco de inferências erradas devidas à evolução do processo ou a faIhas de memória. Uma vez que muitos trabaIhadores só buscam assistência médica meses e até anos após o início dos sintomas, uma maneira de lidar com esse viés seria agrupar os casos quanto ao tempo desde o início dos sintomas, e estudar separadamente cada grupo. Isso permitiria também abordar aspectos como fatores agravantes e de melhoria dos sintomas. 
Uma grande dificuldade em epidemiologia ocupacional é definir e mensurar exposição a fatores potencialmente insalubres. Tal dificuldade existe não apenas pela natureza do fator definido como exposição, no caso das LER posturas, movimentos, organização e equipamentos de trabal ho -, mas também pela concomitância e inter-relação desses fatores e de outros potencialmente importantes como o estresse, o trabal ho sob pressão, a insatisfação no emprego, além de outros individuais.

Tradicionalmente, um fator de exposição é caracterizado a partir da via ou modo de exposição, intensidade, duração, data de início etérmino da exposição. A mensuração e classificação correta da exposição quanto à intensidade e duração permitiriam explorar a existência de uma relação dose-resposta. As doenças abrigadas sob a denominação de LER já trazem embutidas na terminologia o envolvimento de atividade repetitiva, pressupondo na sua própria definição a idéia de uma associação tipo doseresposta. Além disso, a despeito de relatarem uma associação dessas síndromes com movimentos repetitivos, não se verifica na quase totalidade dos estudos revisados, o estabelecimento de gradientes dose-resposta. Nesse sentido, pode-se remeter a uma outra discussão sobre as LER (que não cabe ser aqui aprofundada), relacionada às controvérsias em torno de sua nomenclatura (Cleland, 1987; Armstrong et al., 1993).

Por outro lado, a despeito da insuficiência de conhecimento e métodos diagnósticos dos quadros clínicos englobados como LER, uma série de medidas são tomadas e propostas para prevenir ou tratar os casos existentes. Um aspecto importante para estudo seria a avaliação das medidas e tratamentos propostos como o afastamento do trabalho, fisioterapias, ginásticas e outros. É verdade que a multiplicidade de propostas terapêuticas prescritas isolada e/ ou conjuntamente e a precariedade das informações sobre essas medidas e de acompanhamento das pessoas acometidas dificultam a avaliação da eficácia e especificidade da relação intervenção-resposta. Nesse sentido, os serviços de saúde do trabalhador, juntamente com pesquisadores capacitados, poderiam desenvolver projetos de intervenção, visando avaliar a eficácia de certas medidas propostas e mesmo testar novos procedimentos.

\section{Conclusão}

Os estudos realizados, em sua grande maioria de corte transversal, variam quanto à definição, especificidade e método diagnóstico dos casos estudados e quanto à abordagem de fatores de exposição ocupacional e extra-ocupacional. A despeito dessa diversidade, eles apontam que fatores ligados à ergonomia, organização do trabalho, além de componentes biológico-individuais, como idade, fatores genéticos, gravidez, estado mental, estresses e outras doenças sistêmicas (Kiesler \& Finholt, 1988) estariam envolvidos na gênese das LER.

Todavia esses estudos apresentam limitações metodológicas importantes decorridas da insuficiência e não padronização dos métodos diagnósticos adotados, inclusão de casos incidentes e prevalentes com grau de acometimento variado, falta de transparência nas definições de elegibilidade tanto para casos (expostos) como para controles (ou não expostos), e precariedade na abordagem dos diversos fatores de exposição investigados.

Com relação à exposição os estudos avançam pouco na caracterização dos fatores ocupacionais como esforço, repetitividade e componentes da organização do trabalho como pressões, insatisfação e outros. Não avançam quanto às formas de qualificação e quantificação de sobrecarga no trabalho; por outro lado, os métodos de observação e informações sobre o processo e condições de trabalho, de domínio conexo a diversas disciplinas do conhecimento, não são utilizados em sua totalidade, nem há cruzamento de fontes diversificadas. Além disso, deve-se ressaltar a complexidade e o papel dos aspectos psicológicos, que escapam aos métodos clássicos de apreensão e mensuração, além da dificuldade de se estabelecer sua influência enquanto desencadeante ou conseqüência dos sintomas. Fatores biológicos, como o gênero e a idade, colocam-se, no caso das LER, como possíveis fatores de confusão importantes. Por exemplo, o percentual de mulheres em determinado ramo de atividade é mais alto que o de homens, além das tarefas realizadas variarem para os dois sexos no mesmo ramo.

Parece consensual entre os especialistas da saúde ocupacional a influência das novas tecnologias e exigências de produtividade na gênese das Lesões por Esforços Repetitivos. Porém, do ponto de vista epidemiológico tem sido difícil desdobrar esse conceito em fatores específicos que permitam estimar riscos e ajustes para possíveis elementos de confusão.

Concluindo, o número de trabalhadores acometidos e afastados do trabalho continua a 
crescer. Atitudes extremas de negar ou abraçar acriticamente a existência do problema contribuem pouco para entender esse fenômeno e principalmente para ajudar os trabalhadores e os serviços de saúde ocupacional. Extrapolando as próprias questões metodológicas, as lacunas ainda existentes no conhecimento científico acumulado limitam a avaliação clara das possíveis inter-relações entre tantos possíveis componentes envolvidos na gênese desses sintomas e síndromes abrigados nas LER. Na construção desse saber, vislumbra-se, no momento, a necessidade de investigações específicas e interdisciplinares que tratem com rigor metodológico os desafios apontados para o estudo das LER.

\section{Agradecimentos}

Agradecemos o subsídio parcial da Fundação de Amparo à Pesquisa de Minas Gerais e Secretaria Municipal de Saúde de Belo Horizonte.

\section{Referências}

ARMSTRONG, T. J.; FINE, L. J.; GOLDSTEIN, S. A.; LIFSHITZ, Y. R. \& SILVERSTEIN, B. A., 1987. Ergonomics considerations in hand and wrist tendinitis. Journal of Hand Surgery, 12A:830-837.

ARMSTRONG, T. J.; BUCKLE, P.; FINE, L. J.; HAGBERG, M.; JONSSON, B.; KILBOM, A.; KUORINKA, I. A.; SILVERSTEIN, B. A.; SJOGAARD, G. \& VIIKARIJUNTURA, E. R. A., 1993. A conceptual model for work-related neck and upper limb musculoskeletal disorders. Scandinavian Journal of Work and Environmental Health, 19:73-84.

ASSUNÇÃO, A. A. \& ROCHA, L. E., 1993. Agora até namorar fica difícil: uma história de lesões por esforços repetitivos. In: Vida, Doença eTrabalho no Brasil (J. T. Buschinelli, L. E. Rocha \& R. M. Rigotto, orgs.), pp. 461-471, São Paulo: Vozes.

ASSUNÇÃO, A. A., 1995. Sistema músculo-esquelético: lesões por esforços repetitivos (LER). In: Patologia do Trabalho (R. Mendes, org.), pp. 175181, Rio de Janeiro: Atheneu.

BAMMER, G., 1988. The prevalence of work-related neck and upper limb disorders among office workers in 7 countries: A pilot study. In: Preprints of International Conference on Ergonomics, Occupational Safety and Health and the Environment, Beijing.
BAM MER, G., 1989a. Towards Understanding the Arm Pain Syndrome RSI. University Extension, Course 24. Victoria: University of Western Australia.

BAM MER, G., 1989b. Work-Related Neck and Upper Limb Disorders Associated with OfficeWork. PrevaIence and Causes. Victoria: University of Western Australian.

BAM MER, G., 1990. Review of current knowledge musculoskeletal problems. In: Work with Display Units Conference (L. Berlinguet \& D. Berthelette, eds.), pp. 113-120, Amsterdam: Elsevier Science Publishers B.V.

BAMMER, G., 1996. Work-related neck and upper limb disorders: Social, organisational, biomechanical and medical aspects. In: I Seminário Internacional Fiemg de Saúde Ocupacional/Lesões por Esforços Repetitivos/LER, Belo Horizonte.

BERNARD, B.; SAUTER, S.; FINE, L.; PETERSEN, M . \& HALES, T., 1994. Job task and psychosocial risk factors for work-related musculoskeletal disorders among newspaper employees. Scandinavian Journal of Work and Environmental Health, 20:417-426.

BROWNE, C. D.; NOLAN, B. M. \& FAITHFULL, D. K., 1984. Occupational repetition strain injuries. Guidelines for diagnosis and management. Medical Journal of Australia, 140:329-332. 
CHIANG, H. C.; KO, Y. C.; CHEN, S. S.; YU, H. S.; WU, T. N. \& CHANG, P.Y., 1993. Prevalence of shoulder and upper-limb disorders among workers in the fish-processing industry. Scandinavian Journal of Work Environmental Health, 19:126-131.

CLELAND, L. G., 1987. RSI : a model of social iatrogenesis. Medical Journal of Australia, 147:236-239.

ENGLISH, C. J.; MACLAREN, W. M.; COURT-BROWN, C.; HUGHES, S. P.; PORTER, R. W.; WALLACE, W. A.; GRAVES, R. J.; PETHICK, A. J. \& SOUTAR, C. A., 1995. Relations between upper limb soft tissue disorders and repetitive movements at work. American Journal of Industrial Medicine, 27:75-90.

FERGUSON, D., 1971. An Australian study of telegraphists' cramp. British Journal of Industry Medicine, 28:280-285.

FRANZBLAU, A.; FLASCHNER, D.; ALBERS, J. W.; BLITZ, S.; WERNER, R. \& ARMSTRONG, T., 1993. Medical screening of office workers for upper extremity cumulative trauma disorders. Archives of Environmental Health, 48:164-170.

HIGGS, P. E.; EDWARDS, D. F.; SEATON, M. K.; FEELY, C. A. \& YOUNG, V. L., 1993. Age-related differences in measures of upper extremity impairment. Journal of Gerontology, 48:175-180.

HOCKING, B., 1987. Epidemiological aspects of repetition strain inury in Telecom Australia. Medical Journal of Australia, 147:218-222.

KIESLER, S. \& FINHOLT, T., 1988. The mystery of RSI. American Psychologist, 43:1004-1015.

LUOPAJARVI, T.; KUORINKA, I.; VIROLAINEN, M. \& HOLMBERG, M., 1979. Prevalence of tenosynovitis and other injuries of the upper extremities in repetitive work. Scandinavian Journal of Work and Environmental Health, 5:48-55.

MAEDA, K.; HUNTING, W. \& GRANDJEAN, E., 1982. Factor analysis of localized fatigue complaints of accouting-machine operators. Journal of Human Ergology, 11:37-43.

MCDERM OTT, F. T., 1986. Repetition strain injury: a review of current understanding. Medical Journal of Australia, 144:196-200.

NUSAT/INSS (Núcleo de Referência em Doenças Ocupacionais da Previdência Social), 1995. Relatório Anual. Belo Horizonte: INSS.

OHLSSON, K.; ATTEWELL, R. G.; PALSSON, B.; KARISSON, B.; BALOGH, I.; JOHNSSON, B.; AHLM , A. \&
SKERFVING, S., 1995. Repetitive industrial work and neck and upper limb disorders in females. American Jounal of Industrial Medicine, 27:731747.

ONG, C. N., 1994. Musculoskeletal disorders in operators of visual display terminals. World Health Forum, 15:161-164.

PINHEIRO, T. M. M.; MARTINSJÚNIOR, J. S. \& MARINHO, C. C., 1995. O ADP em dados: perfil da demanda atendida em 1994. Boletim do ADP, 2:6-10.

ROSS, P., 1994. Ergonomic hazards in the workplace: assessment and prevention. AAOHN Journal, 42:171-176.

SANTOS-FILHO, S. B., 1995. Levantamento da Demanda Atendida no Centro de Referência em Saúde do Trabal hador (Cersat). Belo Horizonte: Secretaria Municipal de Saúde.

SOM MERICH, C. M.; M CGLOTHLIN, J. D. \& MARRAS, W. S., 1993. Occupational risk factors associated with soft tissue disorders of the shoulder: a review of recent investigations in the literature. Ergonomics, 36:697-717.

STOCK, S. R., 1991. Workplace ergonomic factors and the development of muscoskeletal disorders of the neck and upper limbs: a meta-analysis. American Journal of Industrial Medicine, 19:87-107.

VANDERPOOL, H. E.; FRIIS, E. A.; SMITH, B. S. \& HARMS, K. L., 1993. Prevalence of carpal tunnel syndrome and other work-related musculoskeletal problems in cardiac sonographers. Journal of Occupational Medicine, 35:604-610.

VORCARO, C. M. R., 1995. Estudo de Morbidade do Ambulatório de Saúde do Trabalhador em Contagem. Curso de Pós-Graduação em Saúde Pública da Universidade Federal de Minas Gerais, Disciplina deSaúdee Trabalho. Belo Horizonte. (mimeo.)

WESTGAARD, R. H.; JENSEN, C. \& HANSEN, K., 1993. Individual and work-related risk factors associated with symptoms of musculoskeletal cmplaints. International Archives of Occupational and Environmental Health, 64:405-413.

WILLIAMS, R. \&WESTM ORLAND, M., 1994. Occupational cumulative trauma disorders of the upper extremity. American Journal of Occupational Therapy, 48:411-420. 